Logic and Political Action in Agustin García Calvo Work

\title{
Lógica y acción política en la obra de Agustín García Calvo
}

Vicente Ordóñez Roig

Universitat Jaume I

vordonez@fis.uji.es

DOI: https://doi.org/10.15366/bp.2020.24.005

Bajo Palabra. II Época. № 24. Pgs: 91-106

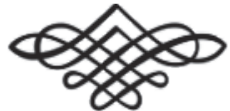


Recibido: 27/10/2019

Aprobado: 28/07/2020

\section{Resumen}

El presente trabajo de investigación pretende ahondar en el pensamiento político de Agustín García Calvo y, en concreto, mostrar la recepción que ha tenido su interpretación de los pensadores presocráticos en la vida política española. La investigación se articula en torno a tres momentos: en primer lugar, se estudia la labor hermenéutica de los pensadores presocráticos y de la lógica que fundamenta su discurso por parte de García Calvo. A continuación, y partiendo del análisis de algunos conceptos clave de García Calvo, se intenta mostrar la influencia que los pensadores presocráticos tuvieron en su pensamiento político. Por último, se investiga el impacto ejercido por alguno de esos conceptos en partidos políticos, sindicatos y movimientos sociales situados a la izquierda del espectro político español.

Palabras clave: Presocráticos, lógica, democracia, dialéctica, ideologías.

\section{Abstract}

This study attempts to deepen the political thinking of Agustín García Calvo and, in particular, to show the reception that his interpretation of the pre-Socratic thinkers has had in Spanish political life. The research is structured around three moments: firstly, I have studied García Calvo hermeneutic work of the pre-Socratic thinkers and the logic that underlies their speech. Secondly, I have tried to show the influence that the pre-Socratic thinkers had in his political thought. Finally, I have analysed the impact of some of these pre-Socratic-inspired concepts on political parties, trade unions and social movements from the left-wing of the Spanish political spectrum.

Keywords: Pre-Socratics, Logic, Democracy, Dialectics, Ideologies. 


\section{Introducción}

EL PRESENTE TRABAJO DE INVESTIGACIÓN quiere profundizar en el pensamiento político de ese crítico obstinado en denunciar las falsedades sobre las que se asienta el orden de lo real que fue Agustín García Calvo. El primer problema con el que me enfrento es que García Calvo no produjo, entre su extensísima bibliografía, una obra de teoría política como tal. Sus reflexiones políticas están diseminadas en libros, conferencias, tertulias, manuscritos o intervenciones en plazas públicas, y uno debe ir tirando del hilo de esa madeja textual con la paciencia y la perspectiva del observador de la naturaleza. La segunda dificultad tiene que ver con el objetivo mismo de mi investigación: mostrar la influencia, muchas veces negativa, que algunos conceptos desarrollados por García Calvo han tenido en partidos políticos, movimientos sociales o sindicatos españoles en los siglos XX y XXI. Es una dificultad importante porque, aunque García Calvo tuvo que exiliarse de España durante el régimen del dictador Franco, estuvo involucrado en las revueltas estudiantiles de los años 60 y en movimientos sociales como el 15-M y simpatizó, además, con diversos colectivos anarquistas, no sólo rechazó las etiquetas políticas, sino que desacreditó las ideologías en su totalidad. Por último, debo referirme a un tercer problema no pequeño que, sin embargo, constituye la clave de bóveda de mi trabajo. Y es que es mi intención mostrar cómo algunos de los conceptos que jalonan el pensamiento político de García Calvo se articulan a partir de su lectura e interpretación de la lógica presocrática: Tales, Anaximandro, Parménides o Zenón, pero sobre todo, de la revisión que realizó de los fragmentos de Heráclito. Trataré de vencer estas tres dificultades a través de un análisis hermenéutico que parte del estudio de un concepto nuclear en la obra de García Calvo (lógica) que, a su vez, remite a una serie de conceptos colindantes y periféricos (pueblo, individuo, poder, mayoría, dialéctica, no, ideologías) que, sostengo, tendrán un eco en agrupaciones, colectivos y sindicatos españoles. Antes, sin embargo, quisiera dilucidar por qué García Calvo se inspira parcialmente en la lógica presocrática para desarrollar algunos de estos conceptos.

\section{Lógica presocrática}

García Calvo entiende la lógica presocrática como una actividad a través de la cual se exploran algunos de los mecanismos del lenguaje y se llevan a sus últimas 
consecuencias estos mecanismos. Desde su punto de vista, la lógica presocrática es pre-filosófica: ni está sometida a la necesidad de sentido, ni está dirigida a la resolución de una cuestión determinada; antes bien, revela las discontinuidades que conforman aquello que nos constituye y pone de manifiesto el carácter esencialmente contradictorio de lo real (Heráclito 10 D-K; Zenón 1 D-K) ${ }^{1}$. No supone, por tanto, doctrina positiva alguna: es una interrogación constante que no trata de llegar a ningún acuerdo o componenda que conecte, anulándolas, las diferencias que a su paso encuentra la razón cuando se lanza a hurgar en la realidad:

'presocrático' ha acabado por venir a querer decir para mí algo como 'prefilosófico' (...), un pensamiento no sumiso todavía a la necesidad de la fe o saber (...), que no parte de la obligación de que este mundo, esto que nos pasa y que somos, sea algo razonable ni posible, y no va desde el principio lastrado y condenado por la finalidad de encontrar solución (o cada vez soluciones más perfectas) a los problemas y contradicciones que el mundo y el lenguaje nos ofrecen, sino sólo animado por un deseo de plantearlos con la honradez y claridad que sea dado ${ }^{2}$.

Para García Calvo, la lógica presocrática se caracteriza por descubrir la (des-) conexión entre mundo y lenguaje, esto es: que en la aprehensión por parte de un sujeto $x$ de un objeto $s$ hay una continuidad que se manifiesta, por una parte, como semantema o término ideal de una lengua y, por otra, como elemento lingüístico delimitado por la sintaxis y la gramática en la que ese término se expresa. Pero esa misma lógica pone de manifiesto que la conexión mundo-concepto es exclusivamente lingüística y que, por muy pequeña que sea la malla terminológica que empleemos, la realidad como tal no se deja apresar por las redes del lenguaje. Esta tensión dialéctica que saca a la luz las contradicciones latentes entre lenguaje y realidad se aprecia, según García Calvo, en numerosos fragmentos presocráticos como, por ejemplo, las pocas citas literales que a través del comentario realizado por Simplicio (Física 140,27) nos han llegado del tratado de Zenón de Elea. En una de las citas Zenón demuestra que, si partimos de la creencia de que hay muchas cosas o que los seres son múltiples (ei polla estin) es necesario, por un lado, que sean todos, i.e., en cantidad finita y definidamente diferentes unos de otros; por otro lado, que no acaben nunca de ser todos, es decir, que sean infinitos en cantidad y no definidamente, sino por interminable gradación de más y menos, diferentes los unos de los otros. García Calvo explica que Zenón trata de hacer evi-

\footnotetext{
${ }^{1}$ García Calvo estudió en profundidad la contradicción lógica en su obra Contra el Tiempo. Para la contradicción en el pensamiento presocrático y en la filosofía en general, cfr. el ensayo de Pleger, W. H., Widerspruch - Identität - Praxis. Argumente zu einer dialektischen Handlungstheorie, Berlín, De Gruyter, 1986, passim.

2 García Calvo, A., Lecturas presocráticas I, Zamora, Lucina, 2001, p. 17.
} 
dente el absurdo de la metodología científica que pretende, de un lado, que hayan conjuntos cerrados formados por un número $x$ de elementos definidos $y$, a la vez, conjuntos abiertos e indefinidos en la medida que entre dos elementos siempre es posible encontrar uno intermedio, y entre un elemento y ese número intermedio otro más, y así sucesivamente 3 .

El desvelamiento de la contradicción fundamental en que nuestra ideación está asentada es un ejemplo de lo que García Calvo pretende recuperar para la reflexión: que la lógica hiriente, que es "lo que para la filosofía (y su Historia) representa el pensamiento presocrático" ${ }^{4}$, vuelva a sonar en público y, a pesar de negar su verdad a las cosas y sus relaciones, ayude a pensar a todo aquel que se atreva a enfrentarse con los problemas desconcertantes que nos acucian. Esta es la tarea que García Calvo hace suya. En sus estudios filológicos, por ejemplo, subraya las disensiones entre lenguaje y realidad por medio de la distinción 'mundo en que se habla' y 'mundo del que se habla's. El mundo en que se habla es el mundo pregramatical o mostrativo, ese ámbito al que los índices deícticos, apuntando desde la producción lingüística, se refieren. Es exterior a la operación lingüística, pero no ajeno, pues de algún modo está relacionado con las operaciones del lenguaje. El mundo del que se habla, por el contrario, es el mundo semántico: el campo de la significación al que las palabras se refieren por su significado. Es platónico, formal, un mundo en el que empleamos un metalenguaje para tratar del campo de los mostrativos -aunque propiamente de ese mundo no puede hablarse, puesto que al hacerlo se le convierte en idea de sí mismo y se le reduce a la significación que de por sí tiene que serle extraña ${ }^{6}$. ¿Cómo se produce, entonces, la juntura o componenda entre ambos mundos? Para García Calvo la fusión es siempre imperfecta porque la realidad surge, no genética, sino poéticamente - es una operación creativa del lógos, del lenguaje o razón común que, al tratar de dar cuenta del campo en que se habla, vive en continua contradicción con su propósito al hablar de él ${ }^{7}$.

\footnotetext{
3 Ibid. p. 53.

${ }^{4}$ García Calvo, A., Lecturas presocráticas II. Razón Común: edición critica, ordenación, traducción y comentario de los restos del libro de Heraclito, Zamora, Lucina, 1985, p.12.

5 La distinción "mundo en / del que se habla" guarda un cierto 'parecido de familia' con el possible world y el context of use de Montague pero es deudora, sobre todo, de la contraposición entre campo deíctico y campo simbólico de Bühler. Cfr. Bühler, K., Sprachtheorie. Die Darstellungsfunktion der Sprache, Stuttgart, Gustav Fischer Verlag, 1982, pp. 79-154; Montague, R., "Universal Grammar", en Formal Philosophy: Selected Papers of Richard Montague (ed. by Richmond H. Thomason), New Haven, Yale University Press, 1974, p. 228.

${ }^{6}$ García Calvo, A., Del lenguaje, Zamora, Lucina, 1979a, p. 341. Podría pensarse que la filosofía logra vencer esta dificultad en conceptos como Dasein, que incluye el índice mostrativo da- (ahí, aquí). No obstante, García Calvo considera que una vez importado a la jerga filosófica, Dasein es inmediatamente sustantivado, es decir: deja de apuntar al campo 'en que' se habla para ser algo 'de lo que' se habla. Cfr. García Calvo, A., Qué es lo que pasa, Zamora, Lucina, 2006, p. 96.

7 García Calvo, A., De Dios, Zamora, Lucina, 1996, p. 136.
} 
Inspirado en la lógica presocrática, García Calvo desarrolla un pensamiento de la contradicción que no niega la verdad de las cosas y sus relaciones, no trata de ocultar ni de armonizar la contradicción viva que late en el fondo de lo real, sino que muestra que esas mismas contradicciones son el fundamento problemático sobre el que se articula la vida del hombre.

\section{Democracia}

LA LÓGICA PRESOCRÁTICA que García Calvo adopta como método para comprender los modos posibles de comportamiento de lo dado pone al descubierto las absurdidades en que la realidad (i.e., sus ideas) está fundada. Al pasar de la lógica a la política — dos órdenes diversos, múltiples, cambiantes según las varias disposiciones de sus partes, pero complementarios- esas mismas contradicciones y absurdidades se harán patentes una vez García Calvo filtre algunos conceptos políticos por su peculiar cedazo crítico.

El primer término que cabe analizar es el de 'democracia'. Para García Calvo, la democracia es la única forma de poder político que se da en los así llamados países desarrollados. Se define por, al menos, dos argucias o falsedades. En primer lugar, hay que reparar en la contradicción que el segundo término hace patente, pues krátos, que significa fuerza o poder, se ejerce necesariamente sobre algo para modificar, deformar o vencer la inercia de ese algo o alguien. Al desempeñar 'demo-' la función de genitivo sujeto de krátos, 'democracia' debe entenderse como la fuerza o poder que ejerce el demos o pueblo. Ahora bien, si es el pueblo el que ejerce la fuerza, ¿contra qué o quién se dirige esa fuerza? ¿ $\mathrm{O}$ es que siendo también los gobernantes pueblo, resulta que los gobernantes no son otros que los gobernados? Según García Calvo, cualquiera con "sentido común sospecha y siente que términos como 'gente' o 'pueblo', después de buscar en vano una definición positiva de su significado, viene a parar en que la sola idea clara que le queda, por lo menos, es una negativa, 'lo que no es el Poder', esto es, 'aquello sobre lo que el Poder se

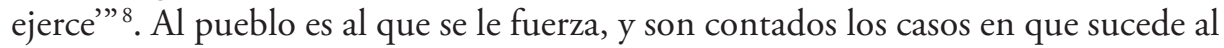
revés, a saber: que sea el pueblo el que tiene la fuerza y la administra. "Sea lo que sea de los avatares de cualquier historia, nunca el pueblo puede tener el poder: el poder está contra el pueblo", clamará en una de sus intervenciones en la asamblea del $15 \mathrm{~m}^{9}$.

${ }^{8}$ García Calvo, A., Qué es lo que pasa, Zamora, Lucina, 2006, p. 124.

9 García Calvo, A., Asamblea de la Puerta del Sol: 19 de mayo, 2011a. Tomado de: http://bauldetrompetillas.es/ agustin-garcia-calvo/tertulias/en-sol/ 
La segunda argucia o falsedad que denuncia está relacionada con el 'demos' democrático: ¿qué es, se pregunta García Calvo, lo que hay por debajo de 'demos-'? Demos, óchlos, turba, populus, son conceptos que en las conjeturas políticas de García Calvo apuntan hacia lo mismo: al pueblo o, como en muchas ocasiones lo denomina recurriendo a una perífrasis que ha tenido cierta repercusión en la nueva izquierda española, a 'los de abajo ${ }^{10}$. El pueblo es de por sí indeterminado, una pluralidad indefinida y, por tanto incontable, no reducible a número fijo. Sin embargo, esa masa indeterminada se convierte en nuestras democracias actuales - aunque no sólo en ellas- en 'conjunto de individuos'. 'Individuo' (individuus) traduce término a término el concepto de la física griega 'átomo': aquello que no se puede cortar ni dividir. El individuo es al demos lo que el átomo al cosmos, a saber, el elemento último e indivisible de una multiplicidad ambigua y porosa por definición. El demos, por tanto, sufre en democracia un desplazamiento: la 'pluralidad indefinida' es reemplazada por la categoría filosófica, política y social de 'individuo', categoría perfilada y claramente definida, representada por un nombre propio, DNI, libro de familia, nacionalidad o profesión. Además, la suma de las opiniones y voluntades individuales constituirá por mayoría una suerte de opinión y voluntad colectiva que será la ley de Todos. Este ardid aritmético — igualar mayoría y totalidad - es el que García Calvo trata de desenmascarar en sus reflexiones sobre la democracia: el paso de una masa indefinida a un conjunto idealmente cerrado compuesto de unidades computables que, por suma o adición, representan aparentemente a la totalidad.

\section{Heráclito como antecedente}

García Calvo considera Que la Denuncia de estas argucias o falsedades de la democracia puede rastrearse en los restos del libro de Heráclito que han llegado hasta nosotros, como se observa en el cuarto de los aforismos de Heráclito que García Calvo recoge en la primera parte de la edición crítica de la obra del pensador de Éfeso, y que se corresponde con el fragmento 2 de la edición Diels-Kranz: "por lo cual hay que seguir a lo público: pues común es el que es público. Pero, siendo la razón común, viven los más como teniendo un pensamiento privado suyo" ${ }^{11}$.

\footnotetext{
${ }^{10}$ García Calvo, A., De la realidad. Conferencia, 1998, p. 18. Tomado de: http://bauldetrompetillas.es/agustin-garcia-calvo/conferencias/. De la polaridad 'arriba'/'abajo' se han reapropiado los partidos políticos surgidos de las movilizaciones del $15 \mathrm{~m}$ que más éxito han alcanzado en la política institucional española: Unidas Podemos y Más País. Cfr. Iglesias, P., “¿Quiénes son los de abajo?”, Diario Público, 8 de julio 2013; Torres, C., "Superando a Iglesias: Errejón se renueva con ecologismo y emulando a Ocasio-Cortez", El Independiente, 29 de septiembre de 2019.

11 García Calvo, A., Lecturas presocráticas II, p. 41.
} 
Probablemente, en ningún otro fragmento puede observarse con tanta nitidez la relación que Heráclito establece entre los pares lógos-xynós y hoi polloi-idios. Ídios, que puede traducirse como 'privado', 'singular' o 'suyo propio', es el individuo particular que tiene una idie phrónesis, una convicción de que su inteligencia es suya y es por ello dueño de lo que piensa, dice y hace ${ }^{12}$. Heráclito menciona al ídios por oposición a lo que denomina xynós: lo común, público y comunitario. ¿Qué es lo común y público? ¿Qué es lo que ni tiene propietario ni dueño, ni nadie que pueda tratar de arrogárselo en exclusividad? En primer lugar, lógos. Cuando légo, que significa originalmente 'reunir', 'recolectar', 'poner junto a' ${ }^{13}$, pasó a designar metafóricamente el lógos, i.e., el 'habla', el 'cálculo' o la 'razón', la lengua griega realizó una gran reflexión sobre sí: al igual que Isis reconstruye los disjecta membra de un Osiris brutalmente despedazado, el lógos reúne a través de la lengua común los jirones de un mundo que, de no mediar él mismo, se perdería inevitablemente. En segundo lugar, la inteligencia o phrónesis. La inteligencia, como el lenguaje, es común a todos y comunitaria (113 D-K), y sólo la pretensión de tener una inteligencia privada vuelve a los hombres extraños e irracionales.

Este mundo - ho mikros, ho megas al que canta Elytis-, a pesar de tener una estructura lógica o lingüística y, por tanto, pública o común, muchos hombres son incapaces de entenderlo. 'Entender', 'saber', son acciones que requieren cordura y moderación (sophrosýne), ese vigor saludable del espíritu caracterizado por el respeto a las leyes y la prudencia (112 D-K). Heráclito parece sugerir que el conjunto de 'los muchos' (hoi polloi), constituido precisamente como conjunto de individuos dotados de una inteligencia particular, está regido por la desmesura y la sinrazón (29 D-K). Sin embargo, los individuos particulares, conscientes y seguros de sí mismos gracias a su superioridad numérica, olvidan que lo que habla por o a través de ellos es el sistema de su lengua, el cual, formando parte de lo preconsciente, se relaciona con la gente indefinidamente plural o común ${ }^{14}$. Quizá por ello afirma Heráclito que los hombres se muestran ininteligentes o necios (axynetoi), incapaces de reconocer la música del lógos aun a pesar de haberla escuchado (1 D-K).

García Calvo considera lógico que Heráclito denuncie la ilusión democrática, "que estima que por sumación de las ideas propias de cada uno se puede, acaso

\footnotetext{
${ }^{12}$ García Calvo señala al respecto que hay que seguir el derivado culto de ídios, 'idiotés', en su penetración en usos populares, donde el individuo particular "ha venido naturalmente a convertirse en idiota, desvelando algo de lo que el pueblo, por lo bajo, siente acerca del asunto”. En García Calvo, A., ¿Qué es lo que pasa?, 126. Lo particular, lo idiótico, es lo contrario de lo común. Para el desarrollo del concepto de individuo en clave presocrática cfr. Salas, I. "A (Very) Brief Doxographical Analysis of Constructivism: from Pre-Socratics to Second-Order Cybernetics”. Bajo Palabra, II Época, No18. 2018, p. 64. DOI: http://dx.doi.org/10.15366/bp2018.18.003

${ }_{13}$ Cfr. Homero Il. 23, 238-239: "recojamos [légomen] después los huesos de Patroclo Menetíada”.

${ }^{14}$ García Calvo, A., Del lenguaje, p. 380.
} 
por virtud del número, producir una inversión del engaño privado en verdad común, cuando parece claro que la suma no hará más que ratificar y robustecer la ilusión individual" ${ }^{15}$. No obstante, ello no implica una defensa de las minorías ni del individuo aislado (49 D-K): ambos casos refuerzan la noción de individuo particular y, por tanto, olvidan que el ser humano tiene su fundamento en lo común a todos.

\section{Dialéctica}

En los escritos de García Calvo de los años '60 y '70 puede rastrearse la influencia del pensamiento presocrático principalmente en su adopción del método dialéctico como instrumento para hacer visibles las contradicciones sobre las que se alza el orden de lo real. Hay que señalar, no obstante, que García Calvo no entiende la dialéctica como un puro método conceptual. Es, además de la estructura de la realidad en su puro mostrarse, una disposición por medio de la cual se descubre la identidad en la contradicción, la definición en la negación, la contradicción en la identidad:

es duro de adquirir y transmitir a otros un método y una disposición dialéctica, esa sensibilidad a las contradicciones de la estructura de la Sociedad y esa habilidad para denunciarlas y derruirlas: es duro, porque así como ello amenaza de verdadera subversión al Orden reinante, así resulta amargo de aprender y perturbador para cada Individuo ${ }^{16}$.

La dialéctica, como actividad que tiene por materia las cosas y las ideas que nos hacemos de ellas, ni puede ser idea alguna ni mucho menos doctrina. Es por esto que García Calvo sostiene que su pensamiento no es un manual de instrucciones o un programa de acción política: hacer esto, indicar qué debe o no hacerse, sería estar ocupando el espacio del poder. Por ello afirma que no puede recomendar hacer tal cosa, pero tampoco recomendar no hacer tal cosa, pues el no hacer queda inmediatamente convertido en un no-hacer que es ya una modalidad del hacer. Lo que sí cabe es sacar a la luz las leyes y órdenes que el orden social vigente —la confusión de Estado-Capital o de Política y Economía - impone: "ayudar a que sus órdenes se vean y se sientan como órdenes. Pues las órdenes del mundo todos las obedecen, pero no todos las oyen" ${ }^{17}$. Esta es una de las pocas tácticas políticas que

\footnotetext{
${ }^{15}$ García Calvo, A., Lecturas presocráticas II, p. 267.

${ }^{16}$ García Calvo, A., Apotegmas sobre el marxismo, Madrid, Luis Manuel Rodríguez Editor, 1979b, p. 11.

${ }^{17}$ Comuna Antinacionalista Zamorana [CAZ], Comunicado urgente contra el despilfarro, Madrid, Luis Manuel Rodríguez Editor, 1977, p. 19.
} 
cabe cultivar, según García Calvo: sacar a la luz, hacer visibles y audibles a través de la palabra en común las instrucciones del Estado-Capital y, en la medida de lo posible, desobedecer las órdenes que, en muchas ocasiones, se confunden con los impulsos íntimos y las voluntades de cada uno.

Para García Calvo la dialéctica es el medio con el que cualquiera puede desocultar las mentiras que se le imponen como única realidad. En esto se filtra de nuevo la influencia del lógos de Heráclito: el lenguaje entendido como una acción en la que la realidad toda se va desenredando y revelando a medida que, al ir razonando, se habla, y justamente con la acción misma de ese modo de hablar que es el razonar. "Todas las cosas se producen según esta razón o lógos" se lee en un extracto del largo fragmento de Heráclito transmitido por Sexto Empírico (1 D-K). Cada uno conforma con su acción lingüística, una suerte de fari fiendo, la operación del lógos, que es común. De ahí la reticencia de García Calvo a que su voz sea tomada con carácter particular y ejemplar, y su insistencia en que su palabra sea escuchada como una más entre otras, una que se diluye entre las muchas voces que configuran la realidad. "Dejar que ese hablar que nos hemos aquí traído sea un hacer él solo y por sí mismo, y confiar (sin garantía alguna, desde luego) en que, si algo pueden hacer las palabras para modificar las actitudes de los sujetos y dificultar el dominio del Estado, ya lo habrán hecho con sólo que se las haya oído" ${ }^{18}$. Esta es la humilde tarea política que cabe llevar a cabo: hablar razonando, que es ya un hacer o producir.

García Calvo desechará explícitamente cualquier estrategia que intente alterar el statu quo por diversos medios: tratar de extraer conclusiones prácticas de un discurso teórico, pretender dar respuesta al inevitable ¿qué hacer?, es caer en la trampa. Los discursos de este tipo conforman esquemas ideológicos, son esencialmente integradores y reaccionarios y, sobre todo, apuntan al establecimiento de unos fines supuestamente ideales. "No sólo rechazamos desde aquí toda idea de independencia entre los medios y los fines, sino que desde aquí anunciamos formal y negativamente que no creemos en que haya fines, sino sólo, en todo caso, medios" ${ }^{19}$. Según García Calvo, los fines están en la estructura misma de los medios y en los instrumentos que se fabrican para conseguirlos y constituye, por tanto, una falacia "el pensar que las armas del Enemigo (que está necesariamente inscrito Él mismo en la forma de sus armas) pueden usarse contra el Enemigo" ${ }^{20}$. Esta con-

\footnotetext{
18 García Calvo, A., Qué es el Estado, Barcelona, La Gaya Ciencia, 1977, p. 71.

19 Comuna Antinacionalista Zamorana [CAZ], Manifiesto de la Comuna Antinacionalista Zamorana, Madrid, Luis Manuel Rodríguez Editor, 1978, p. 60.

${ }^{20}$ García Calvo, A., Qué es el Estado, p. 69.
} 
vicción le llevará a atacar cualquier ideología, tanto las liberales y conservadoras como sus contrarias.

\section{No}

Fuera del Ámbito académico, García Calvo desarrolló buena parte de su actividad pública en ateneos y centros vinculados al sindicato CNT, a pesar de no estar él mismo afiliado ni pertenecer a colectivo o sindicato anarquista alguno. En el anarquismo García Calvo encontrará interlocutores con los que hablar y será, en parte, en medios libertarios que ensaye esa práctica constante del lógos o razón común ${ }^{21}$. Esta proximidad con el anarquismo, sin embargo, no hizo que el pensador zamorano dejara de indicar los desajustes que estaban a la base de su proyecto, empezando por la partícula negativa an-que incorpora el propio término. 'No' es, para García Calvo, la voz de la razón común, la voz de la protesta, una de las pocas herramientas con las que el pueblo puede destruir las ideas que se desarrollan para defender la realidad. Incorporada como negación de la autoridad o poder (arché), el concepto 'anarquía' se carga de significado, se convierte en algo positivo y deja de servir para la crítica de esa realidad incierta llamada política ${ }^{22}$. ¿Por qué García Calvo defiende que ese 'no' que opera dentro del anarquismo es anulado y asimilado? Porque el anarquismo propone un proyecto soteriológico que, como tal, no puede sino estar orientado hacia el futuro. Esa proyección hace que el anarquismo se sirva de los mismos procedimientos y medios organizativos que el poder que niega utiliza, transformándose en una logomaquia o derivando incluso en prácticas demagógicas ${ }^{23}$. La negación queda así domesticada: "guardarse de ese sometimiento del No a alguna especie de plan futuro como el de los de Ellos, el de los que mandan, es elemental, es de la cosa más elemental que en cuestión de política se puede decir" 24 . La simple negación, decir 'no' a lo que pasa no tiene significado y, por tanto, no puede integrarse en sistema alguno:

\footnotetext{
${ }^{21}$ García Calvo, A., "Lo único apreciable de la universidad son los locales". Bicicleta: Revista de comunicaciones libertarias, 23-24, 68-69, 1980, p. 69.

22 García Calvo, A., Carta a Carlos Semprún y Javier Domingo: contra la idea de hacer la Historia del Anarquismo. Historia Libertaria, no 1, 1978. Para la conexión entre política e incertidumbre, cfr. Campillo, A., "Hacia un nuevo concepto de lo político". Bajo Palabra II Época, 20, 2019, p. 35. DOI: http://doi.org/10.15366/ bp2019.20.0018

${ }_{23}$ Véase al respecto Brecher, B. y Ordóñez, V., "Narratives of Power: Demagogues, Politics and Morality at the Start of the 21st Century". Araucaria. Revista Iberoamericana de Filosofia, Politica, Humanidades y Relaciones Internacionales, vol. 21, n 42, 2019, p. 478.

${ }^{24}$ García, M.C. y Nacarino, P., “Contra la Realidad: entrevista a Agustín García Calvo”. Periódico CNT, no 324, 2006, p. 21.
} 
decir 'no' al Poder, al Estado, al Capital, al Dinero, a la Familia, en especial al Régimen que hoy padecemos, en el cual culminan todos los regímenes de la Historia y donde esa labor funesta que he descrito se da de la manera más perfecta: en la democracia desarrollada que se funda en la fe en el individuo personal; y, por tanto, decir 'no' - y esto es lo más difícil y más importante, — decir 'no' a la persona de cada uno, en contra de lo que manda la democracia ${ }^{25}$.

Decir 'no' es aceptar 1) la negación como origen de la lengua y, por tanto, del ser; 2) la contradicción como forma de la realidad — en la medida en que cada cosa se obtiene por negación de otra ${ }^{26}$. La negación es la consecuencia lógica del descubrimiento de la falsedad de la realidad, de que la realidad no es lo que es. Cabe añadir, además, que ese sencillo 'no' con el que García Calvo intenta echar abajo la fe en el orden de lo real no ofrece nada a cambio, no pone otra cosa en su lugar, sino que lleva la contradicción y la guerra contra el poder hasta sus últimas consecuencias y posibilita que surja lo que no se sabe: la negación aparece como el mecanismo primario de la lengua o lógica común.

La influencia de este 'no' de inspiración presocrática con el que García Calvo se enfrenta a lo que está establecido como real puede palparse en diversas manifestaciones políticas, sociales y culturales. Dentro del anarquismo, simpatizantes anarcosindicalistas, militantes y afines a la CNT han mostrado cierta sintonía con la actitud política de García Calvo, defendiendo que las ideologías desempeñan diversas funciones dentro de la compleja maquinaria que asegura el control social incluida la manufactura de la mentira. La única táctica política legítima consistiría, para ellos, en decir 'no': no al futuro; no al progreso; no a la democracia representativa; no a ningún '-ismo', incluido el anarquismo ${ }^{27}$. Por otra parte, si bien activistas de movimientos ciudadanos como el $15 \mathrm{~m}$ apoyaron a García Calvo en ese 'no' total en el que cifraba buena parte de su lucha política, hay que añadir que esos activistas representaban al sector más radical y minoritario dentro del movimiento ${ }^{28}$. Cabe señalar, por último, el ascendente de este 'no' en artistas que, como Chicho Sánchez Ferlosio, Isabel Escudero o Santiago Sierra, han desarrollado una actitud hipercrítica con los aparatos e instituciones sociales —Estado, Iglesia, sistema económico y poderes políticos.

\footnotetext{
${ }_{25}$ García Calvo, A. y Escudero, I., ¿Quién dice No? En torno a la anarquía, Madrid, Fundación Estudios Libertarios Anselmo Lorenzo, 1999, p. 14.

${ }_{26}$ García Calvo, A., Heráclito y la lógica. Conferencia, Madrid, 1991, p. 4. Tomado de: http://bauldetrompetillas. es/agustin-garcia-calvo/conferencias/

27 García Calvo, A. y Escudero, I., ¿Quién dice No? En torno a la anarquía, p. 31.

${ }^{28}$ Cfr. Ruiz Fernández, J., "Agustín García Calvo en el 15-M”. Las Torres de Lucca, no 8, 2016, p. 273; Ordóńez, V., "Agustín García Calvo in Our Time”. Radical Philosophy, 2.03, Diciembre 2018, p. 76.
} 


\section{Conclusiones}

El pensamiento en acción de García Calvo representa el intento por revitalizar la obra de los filósofos presocráticos en el ámbito occidental desde una perspectiva lógica, pero también política y social. A diferencia de quienes en nuestro tiempo ofrecen una serie de saberes o verdades irrefutables al amparo de las ciencias positivas, la lógica de García Calvo no ofrece saber de ningún tipo, ni particular ni absoluto. García Calvo se dedica, por un parte, a denunciar los engaños que configuran la realidad; por otra, a llamar la atención sobre la progresiva disolución del sentido y la razón común. Después de la pregunta y la contradicción dialéctica que pone en cuestión los hechos y las cosas todas, no cabe para el pensador zamorano sino adoptar la actitud de Heráclito y combatir negativamente las ideas que apuntalan una realidad siempre aparente. ¿Significa esto que el pensamiento de García Calvo es un pensamiento teorético alejado de los problemas comunes? Ciertamente no. Su discurso se entrecruza con unas circunstancias históricas y unas luchas sociales muy concretas. Por ello, porque vivió a caballo entre la dictadura franquista y la democracia española, la obra de García Calvo tiene un marcado cariz político. En ella trata de responder de algún modo a la pregunta que indaga sobre las posibilidades de llevar una vida buena: "un hombre bueno para la razón no puede ser sino aquél que, en virtud de una cierta conciencia de su propia contradicción constitutiva, participa algo menos en la creencia en su idie phrónesis o sabiduría particular, y resulta por ello algo más racional y comunitario" ${ }^{29}$. Ahora bien, ¿no cabe hacer nada más? ¿Toda la propuesta política de García Calvo se agota en esa renuncia? ¿Ni elabora un manual de instrucciones ni propone un ideario a partir de su caja de herramientas conceptual? ¿Sólo cabe decir 'no'? Efectivamente, las críticas a su pensamiento político irán casi siempre en esta dirección. Sin embargo, García Calvo no cederá a la tentación de ofrecer programa alguno y argumentará que en la renuncia a uno mismo y en el 'no' se cifra casi todo lo que cabe hacer: el 'no' es el corazón del lenguaje y el corazón del pueblo.

En este contexto cabe ubicar su crítica al entramado desde el cual se articula el sistema democrático. Como hemos tenido ocasión de comprobar, en sus escritos y actos públicos sostuvo que la democracia se asentaba sobre una serie de falsedades que hacían de ella el sistema que mejor servía a los intereses del binomio Estado-Capital. Es por ello, quizá, que García Calvo se sintió más cómodo entre grupos de estudio y agitación libertarios, movimientos sociales críticos con el statu quo o iniciativas marginales como la ocupación de edificios o la creación de es-

${ }^{29}$ García Calvo, A., Lecturas presocráticas II, p. 267. 
cuelas populares. Fenómenos espontáneos como las revueltas estudiantiles de los años '60 o la toma de espacios públicos que tuvieron lugar tras el 15M fueron para García Calvo ejemplos de cómo robustecer el asociacionismo en la sociedad civil, el asamblearismo, la democracia directa y la autoorganización de los afectados por las injusticias cometidas desde el poder.

De Agustín García Calvo bien podría afirmarse que fue, como Zenón de Elea, un pánton epiléptor o crítico de las cosas todas empeñado en denunciar las falsedades sobre las que se asienta la realidad. Esa realidad, sin embargo, deja abiertas grietas y puntos de fuga que pueden ser detectadas a través de eso público, gratuito y comunitario que llamamos lenguaje y que García Calvo identifica con el lógos o razón común. 


\section{REFERENCIAS Bibliográficas}

Brecher, B. y Ordóńez, V., "Narratives of Power: Demagogues, Politics and Morality at the Start of the 21st Century". Araucaria. Revista Iberoamericana de Filosofia, Politica, Humanidades y Relaciones Internacionales, vol. 21, n 42, 2019, p. 478.

Bühler, K., Sprachtheorie. Die Darstellungsfunktion der Sprache, Stuttgart, Gustav Fischer Verlag, 1982.

Campillo, A., "Hacia un nuevo concepto de lo político". Bajo Palabra II Época, 20, 2019.

Comuna Antinacionalista Zamorana [CAZ], Comunicado urgente contra el despilfarro, Madrid, Luis Manuel Rodríguez Editor, 1977.

Comuna Antinacionalista Zamorana [CAZ], Manifiesto de la Comuna Antinacionalista Zamorana, Madrid, Luis Manuel Rodríguez Editor, 1978.

Diels, H. y Kranz, W., Die Fragmente der Vorsokratiker, Berlín, Weidermann, 1952.

García, M.C. y Nacarino, P., "Contra la Realidad: entrevista a Agustín García Calvo". Periódico CNT, no 324, 2006.

García Calvo, A., Qué es el Estado, Barcelona, La Gaya Ciencia, 1977.

García Calvo, A., Carta a Carlos Semprún y Javier Domingo: contra la idea de hacer la Historia del Anarquismo. Historia Libertaria, no 1, 1978.

García Calvo, A., Del lenguaje, Zamora, Lucina, 1979a.

García Calvo, A., Apotegmas sobre el marxismo, Madrid, Luis Manuel Rodríguez Editor, 1979b.

García Calvo, A., "Lo único apreciable de la universidad son los locales". Bicicleta: Revista de comunicaciones libertarias, 23-24, 68-69, 1980.

García Calvo, A., Lecturas presocráticas II. Razón Común: edición critica, ordenación, traducción y comentario de los restos del libro de Heraclito, Zamora, Lucina, 1985.

García Calvo, A., Avisos para el derrumbe, Zamora, Lucina, 1988.

García Calvo, A., Heráclito y la lógica. Conferencia, Madrid, 1991. Tomado de: http://bauldetrompetillas.es/agustin-garcia-calvo/conferencias/ 
García Calvo, A., Contra el Tiempo, Zamora, Lucina, 1993.

García Calvo, A., De Dios, Zamora, Lucina, 1996.

García Calvo, A., Contra el Hombre, Madrid, Fundación Estudios Libertarios Anselmo Lorenzo, 1997.

García Calvo, A., De la realidad. Conferencia, 1998. Tomado de: http://bauldetrompetillas.es/agustin-garcia-calvo/conferencias/

García Calvo, A. y Escudero, I., ¿Quién dice No? En torno a la anarquía, Madrid, Fundación Estudios Libertarios Anselmo Lorenzo, 1999.

García Calvo, A., Lecturas presocráticas I, Zamora, Lucina, 2001.

García Calvo, A., Qué es lo que pasa, Zamora, Lucina, 2006.

García Calvo, A., Ateneo de Madrid. Tertulia Política no 80, 2007. Tomado de: http://bauldetrompetillas.es/agustin-garcia-calvo/tertulias/en-el-ateneo/

García Calvo, A., Asamblea de la Puerta del Sol: 19 de mayo, 2011 a. Tomado de: http://bauldetrompetillas.es/agustin-garcia-calvo/tertulias/en-sol/

García Calvo, A., Ateneo de Madrid. Tertulia Política no 283, 2011b. Tomado de: http://bauldetrompetillas.es/agustin-garcia-calvo/tertulias/en-el-ateneo/

García Calvo, A., Asamblea de la Puerta del Sol: 16 de junio, 2011c. Tomado de: http://bauldetrompetillas.es/agustin-garcia-calvo/tertulias/en-sol/

Montague, R., Formal Philosophy: Selected Papers of Richard Montague (ed. by Richmond H. Thomason), New Haven, Yale University Press, 1974.

Ordóñez, V., "Agustín García Calvo in Our Time". Radical Philosophy, 2.03, 2018.

Pleger, W. H., Widerspruch - Identität - Praxis. Argumente zu einer dialektischen Handlungstheorie, Berlín, De Gruyter, 1986.

Ruiz Fernández, J. (2016), "Agustín García Calvo en el 15-M". Las Torres de Lucca, no 8, 247-279.

Salas, I. "A (Very) Brief Doxographical Analysis of Constructivism: from Pre-Socratics to Second-Order Cybernetics". Bajo Palabra, II Época, No18. 2018, p. 64. DOI: http://dx.doi.org/10.15366/bp2018.18.003

DOI: https://doi.org/10.15366/bp.2020.24.005

Bajo Palabra. II Época. No 24. Pgs: 91-106 